\title{
Optical and Structural Characterization of InGaN/GaN Multiple Quantum Wells by Epitaxial Lateral Overgrowth
}

\author{
Masakazu Sugiyama ${ }^{1}$, Tomonari Shioda ${ }^{2, *}$, Yuki Tomita ${ }^{2, *}$, \\ Takahisa Yamamoto $^{3}$, Yuichi Ikuhara ${ }^{1}$ and Yoshiaki Nakano ${ }^{4}$ \\ ${ }^{1}$ Institute of Engineering Innovation, School of Engineering, the University of Tokyo, Tokyo 113-8656, Japan \\ ${ }^{2}$ Department of Electrical Engineering and Information Systems, School of Engineering, the University of Tokyo, \\ Tokyo 113-8656, Japan \\ ${ }^{3}$ Department of Advance Materials Science, School of Frontier Sciences, the University of Tokyo, Kashiwa 277-8561, Japan \\ ${ }^{4}$ Research Center for Advanced Science and Technology, the University of Tokyo, Tokyo 153-8904, Japan
}

In order to examine the effect of threading dislocations on the structure of InGaN/GaN multiple quantum wells (MQWs) grown by metalorganic vapor-phase epitaxy (MOVPE), epitaxial lateral overgrowth (ELO) on a patterned sapphire substrate was employed and the MQWs were characterized as a function of the lateral position in terms of cathode luminescence (CL), transmission electron microscopy (TEM) and scanning transmission electron microscopy (STEM). The intensity of a blue luminescence peak $(426 \mathrm{~nm})$ was larger for the epitaxial layer overriding on the masks than that for the layer on the unmasked area, while the peak wavelength was independent of the position. Threading dislocations, which were generated at the GaN/sapphire interface, did not propagate to the surface of GaN layer on the masks. The thicknesses of both the InGaN well and the GaN barrier, on the other hand, were the same for the MQWs both on the unmasked surface and on the masks, which is consistent with the invariable peak wavelength of the blue luminescence. For an InGaN well with the indium content of around $10 \%$, it seems that the existence of threading dislocations does not affect the structure of the MQWs but just reduces the luminescence intensity through a recombination via mid-gap states. [doi:10.2320/matertrans.MC200830]

(Received December 8, 2008; Accepted February 13, 2009; Published April 8, 2009)

Keywords: InGaN, multiple quantum wells (MQWs), metal-organic vapor-phase epitaxy (MOVPE), epitaxial lateral overgrowth (ELO)

\section{Introduction}

There is an increasing demand for solid-state lighting using light-emitting diodes (LEDs). $\operatorname{In}_{x} \mathrm{Ga}_{1-x} \mathrm{~N}$ is a promising material for the active medium of LEDs because the bandgap of $\operatorname{In}_{x} \mathrm{Ga}_{1-x} \mathrm{~N}$ varies from 0.65 to $3.6 \mathrm{eV}$ as a function of an indium content $x$, which covers from infrared to ultraviolet wavelength range. $\operatorname{In}_{x} \mathrm{Ga}_{1-x} \mathrm{~N}$ is also free from hazardous elements.

Growth of $\operatorname{In}_{x} \mathrm{Ga}_{1-x} \mathrm{~N}$ has confronted a lot of difficulties. Since an $\mathrm{In}_{x} \mathrm{Ga}_{1-x} \mathrm{~N}$ layer is typically grown on a $\mathrm{GaN}$ underlayer, there is compressive strain on the $\operatorname{In}_{x} \mathrm{Ga}_{1-x} \mathrm{~N}$ layer which arises difficulties in both the coherent growth of the $\operatorname{In}_{x} \mathrm{Ga}_{1-x} \mathrm{~N}$ and the incorporation of indium by a large amount. Due to this strain, it is difficult to grow a thick $\mathrm{In}_{x} \mathrm{Ga}_{1-x} \mathrm{~N}$ layer with a uniform indium content. Instead, multiple quantum wells (MQWs) containing thin (a thickness of $1-10 \mathrm{~nm}$ ) $\operatorname{In}_{x} \mathrm{Ga}_{1-x} \mathrm{~N}$ layers are commonly used. MQWs are also beneficial for carrier confinement that increases luminescence intensity.

Along with the difficulty in the growth of InGaN itself, a GaN underlayer has been accompanied by the difficulties in the growth on a foreign substrate. Since the bulk growth of $\mathrm{GaN}$ is difficult, GaN layers have been grown on sapphire substrates, which induces numerous dislocations in the GaN layers due to the lattice mismatch between $\mathrm{GaN}$ and sapphire. Such dislocations significantly affect the structure of InGaN/ GaN MQWs; especially, lateral uniformity of both the thickness and the indium content of the InGaN wells can be deteriorated by the existence of dislocations. ${ }^{1-5)}$ Even if the structure of the MQWs is unaffected by dislocations, trans-

*Graduate Student, The University of Tokyo port properties of electrons and holes, which are often clearly manifested in the properties such as luminescence wavelengths and their intensities, can be affected by the existence of dislocations. ${ }^{4)}$

For the system with a large mismatch between an epitaxial layer and a substrate, epitaxial lateral overgrowth (ELO), in which a crystal layer is grown on a patterned substrate not only to vertical but also to lateral directions, can provide an epitaxial layer without dislocations on the masked area because threading dislocations stemming from the interface between the layer and the substrate do not propagate in the lateral direction. ${ }^{6,7)}$ In other words, the ELO can provide the epitaxial layer that has a significant distribution of the dislocation density in a way that is defined by the mask shape on a substrate.

The aim of this work is to clarify the effect of threading dislocations on the structure and the optical properties of InGaN/GaN MQWs. For this purpose, a $\mathrm{GaN}$ template grown by the ELO is desirable because it has a designed distribution in the dislocation density. By the growth of the MQWs on the ELO template, we can obtain the MQWs affected by the threading dislocations and those without such an effect simultaneously, keeping the other conditions exactly the same. For the characterization of luminescence properties, cathode luminescence was employed because it provides spatially-resolved luminescence spectra of a layer with a resolution of approximately $0.1 \mu \mathrm{m}$. For the structural characterization of the MQWs, cross-sectional transmission electron microscopy (TEM) was employed. Along with conventional bright-field TEM images, scanning TEM (STEM) was employed to observe the threading dislocations and the structure of MQWs more clearly as decoupled from the contrast due to the strain of a sample. 

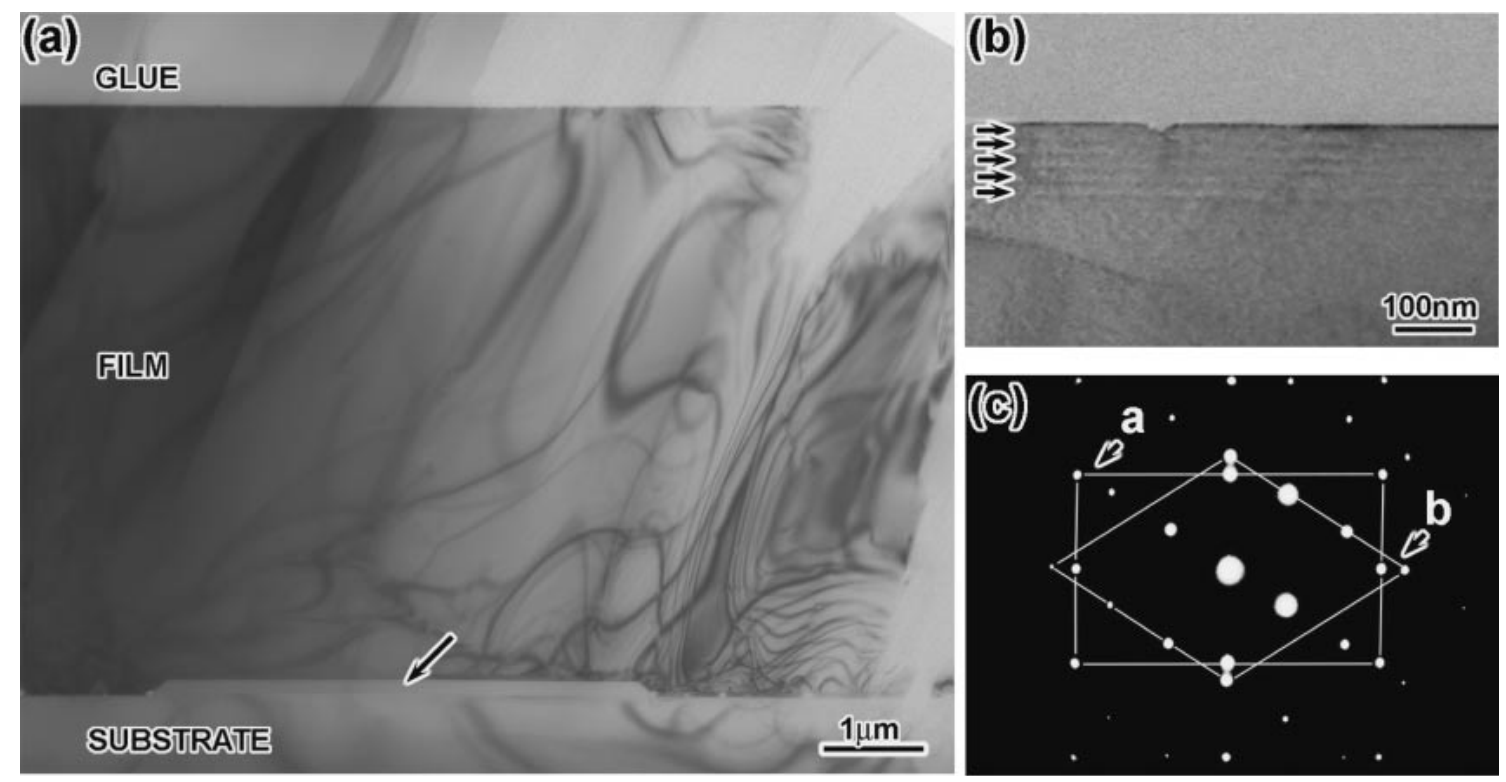

Fig. 1 (a) A bright-field cross-sectional TEM image of the GaN layer on a patterned sapphire substrate. The arrow indicates a mask on the sapphire substrate. (b) A magnified image at the surface of the layer. Five InGaN quantum wells exist in the vicinity of the surface. (c) A selected area diffraction (SAD) pattern at the interface between the sapphire substrate and the GaN layer. The rectangle "a" indicates the spots from a GaN layer and the diamond "b" for a sapphire substrate, indicating that the cross sections are m-plane (1100) for the GaN layer and the a-plane (1120) for the sapphire substrate, respectively.

\section{Experimental}

\subsection{Growth of InGaN/GaN MQWs}

For the epitaxial growth of $\mathrm{GaN}$ and $\mathrm{InGaN}$, metal-organic vapor-phase epitaxy (MOVPE) was adopted because it is one of the most suitable methods for both the ELO and the growth of MQWs, especially for nitride semiconductors. A 2.3- $\mu \mathrm{m}$ thick GaN layer was grown by MOVPE on a patterned 2-inch sapphire substrate, yielding a flat template for the growth of the InGaN/GaN MQWs. This template was once cleaved to make smaller test substrates, and then the substrate was introduced to the MOVPE reactor to grow the InGaN/GaN MQWs. The detailed experimental conditions are described below.

A $0.1-\mu$ m-thick $\mathrm{SiO}_{2}$ film was deposited on a (0001) sapphire substrate by sputtering. A line-and-space pattern, consisting of a repetition of a mask and a spacing, was patterned along the [11 20$]$ direction of the substrate using photolithography and wet-chemical etching. Since the [1100] direction of $\mathrm{GaN}$ is parallel to the [1120] direction of the sapphire substrate, as described later in the section 3.1, the stripe pattern is along the [1100] direction of GaN.

The patterned substrate was introduced to an MOVPE reactor (Aixtron, AIX 200/4 RF-S), and was heated at $1175^{\circ} \mathrm{C}$ under $\mathrm{H}_{2}$ flow for thermal cleaning. The growth of $\mathrm{GaN}$ started from the low-temperature buffer growth at $550^{\circ} \mathrm{C}$ and then high-temperature continuous growth was executed for $45 \mathrm{~min}$ at $1200^{\circ} \mathrm{C}$. The total pressure was $2 \times 10^{4} \mathrm{~Pa}$ and the source materials were $\left(\mathrm{CH}_{3}\right)_{3} \mathrm{Ga}(\mathrm{TMGa})$ and $\mathrm{NH}_{3}$. The growth of the $\mathrm{GaN}$ layer starts from the unmasked sapphire surface, as confirmed by the analysis of initial nucleation, and then the GaN layer grows not only in the vertical direction but also in the lateral direction to form a $\mathrm{GaN}$ layer overriding on the masks. This is a typical epitaxial lateral overgrowth (ELO).
The growth of the MQWs consisting of InGaN well and GaN barrier layers were grown in the different growth batch from the underlying $\mathrm{GaN}$ template. After the cleaved template was cleaned by $\mathrm{H}_{2} \mathrm{SO}_{4}$ and $\mathrm{DI}$ water, it was introduced to the same MOVPE reactor and a 3-nm-thick GaN buffer layer, 5 pairs of MQWs consisting of a 3-nmthick InGaN well and a 17-nm-thick GaN barrier, and a 17nm-thick $\mathrm{GaN}$ cap layer were grown at $800^{\circ} \mathrm{C}, 2 \times 10^{4} \mathrm{~Pa}$. The precursors were $\left(\mathrm{C}_{2} \mathrm{H}_{5}\right)_{3} \mathrm{Ga}$ (TEGa), $\left(\mathrm{CH}_{3}\right)_{3} \mathrm{In}$ (TMIn) and $\mathrm{NH}_{3}$ with $\mathrm{N}_{2}$ carrier. An indium content in the InGaN well was $0.10 \pm 0.02$ as estimated by the fringes of X-ray diffraction $(2 \theta-\omega)$. The MQWs are not doped.

\subsection{Characterization}

For the optical characterization of the MQWs, cathode luminescence (CL) (JEOL, JSM-7000F) was used for the measurement of spatially-resolved luminescence. In this measurement, an acceleration voltage was $15 \mathrm{kV}$, and absorption current was $3 \times 10^{-10} \mathrm{~A}$. Spatial resolution is determined by the diffusion length of a minority carrier and is roughly several ten nanometers. Carbon was sputtered on the surface of the sample to prevent the charge-up.

For the structural characterization, cross-sectional TEM and STEM were adopted. Thin foils for TEM and STEM observation were prepared by the conventional method of grinding, dimpling and finally Ar ion milling. TEM and STEM observation were conducted by a field emission type high resolution TEM (TOPCON, EM-002BF) and a Cscorrected STEM (JEOL, JEM-2100F), respectively.

\section{Results and Discussion}

3.1 Epitaxial lateral overgrowth characterized by TEM

Figure 1(a) shows the cross section of the GaN layer on the patterned sapphire. The cross section is perpendicular 


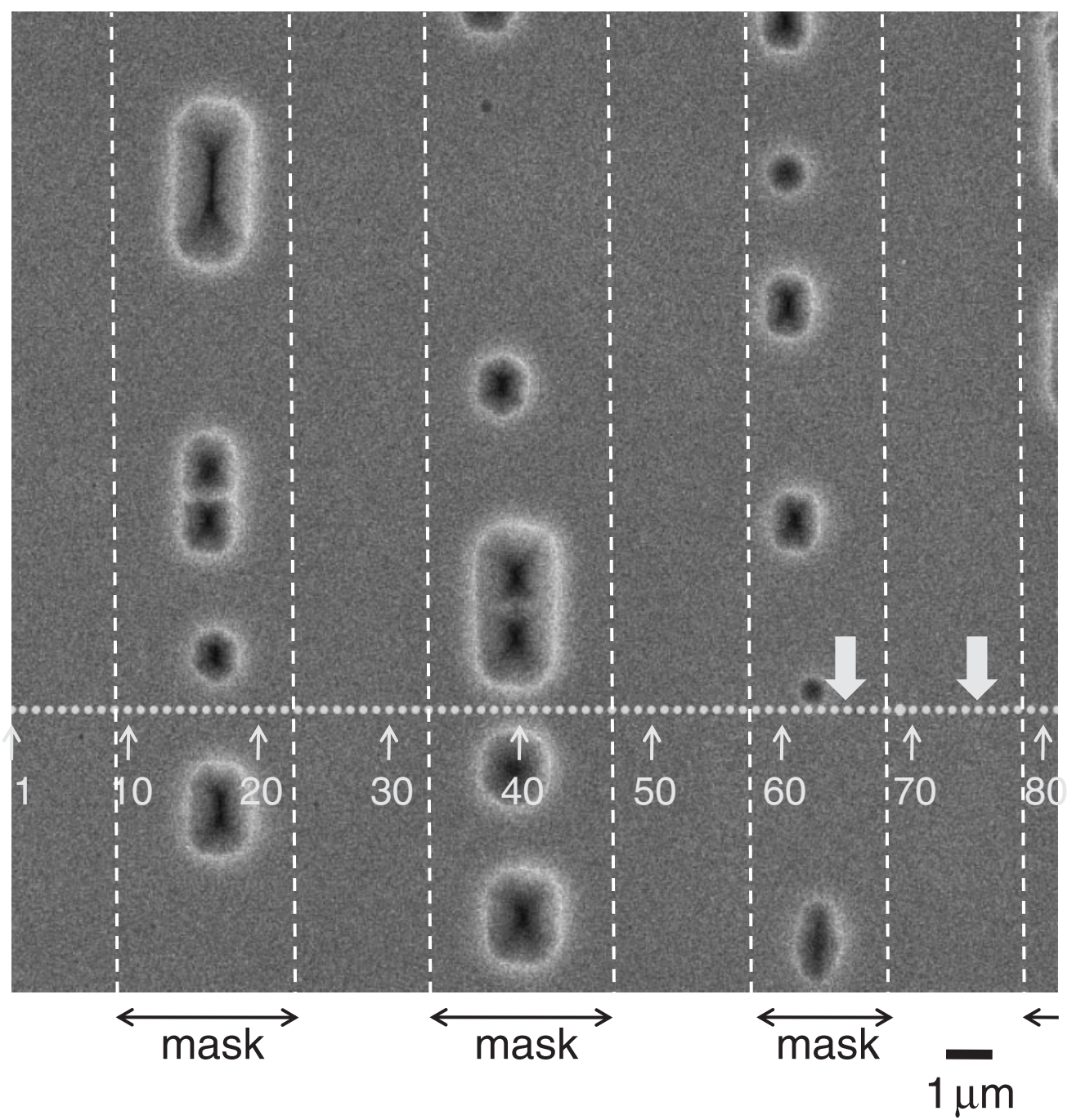

Fig. 2 A top-view SEM image of the sample. The dashed lines indicate the position of the masks beneath the GaN layer. The dots indicate the positions of cathode luminescence measurement, with the numbers indicating the index of the position. The pits exist on the center of a mask where the GaN layers growing laterally from the both edges of the mask failed to merge. The two bold arrows show the positions corresponding to the spectra in Fig. 3.

to the stripe mask patterns. The growth of the GaN layer starts on the unmasked sapphire surface and the layer overrides on the mask without voids on it, resulting in a 5.8$\mu \mathrm{m}$ thick planar layer. The surface of the layer was almost flat, but there sometimes existed pits at the center of the mask where two GaN layers from the both mask edges collide with each other. A selected area diffraction (SAD) pattern taken at the interface between the GaN layer and the sapphire substrate, as shown in Fig. 1(c), indicates that the cross sections are the m-plane (1100) for the GaN layer and the a-plane $(11 \overline{2} 0)$ for the sapphire substrate, respectively. Since two diffraction patterns can be clearly observed at the same time, the GaN layer grows epitaxially on the sapphire substrate with a rotation angle of $30^{\circ}$, which is a typical relationship for the epitaxial growth on the c-plane (0001).

On the top of the GaN layer by ELO, 5 periods of InGaN/ GaN MQWs were grown successfully on the flat c-plane $\mathrm{GaN}$ underlayer, as indicated by the arrows in Fig. 1(b). The spacing of the wells is confirmed to be $20 \mathrm{~nm}$.

\subsection{Cathode luminescence from InGaN/GaN MQWs and GaN underlayers}

Figure 2 shows the top-view scanning electron microscope (SEM) image of the InGaN/GaN MQWs. As shown by the dashed lines, the positions of the mask edges are estimated from the mask pattern and the position of the pits which mostly existed at the center of the masks. Note that the micrometer-scale pits are formed by inadequate coalescence of the layers growing in the lateral direction from the unmasked areas and do not correspond to the position of threading dislocations. The following spatially-resolved cathode luminescence analysis concerns the 81 points as shown in the figure.

Figure 3 shows the cathode luminescence spectra observed at the positions 65 and 75 in Fig. 2, as indicated by the bold arrows. Three typical luminescence peaks were observed: $366 \mathrm{~nm}$ for the emission from GaN bulk, $426 \mathrm{~nm}$ for the emission from the InGaN well, and $562 \mathrm{~nm}$ which is called 'yellow luminescence' and is associated with defect states between $\mathrm{GaN}$ band edges. ${ }^{8-10)}$ The spectrum at the point 65 , 


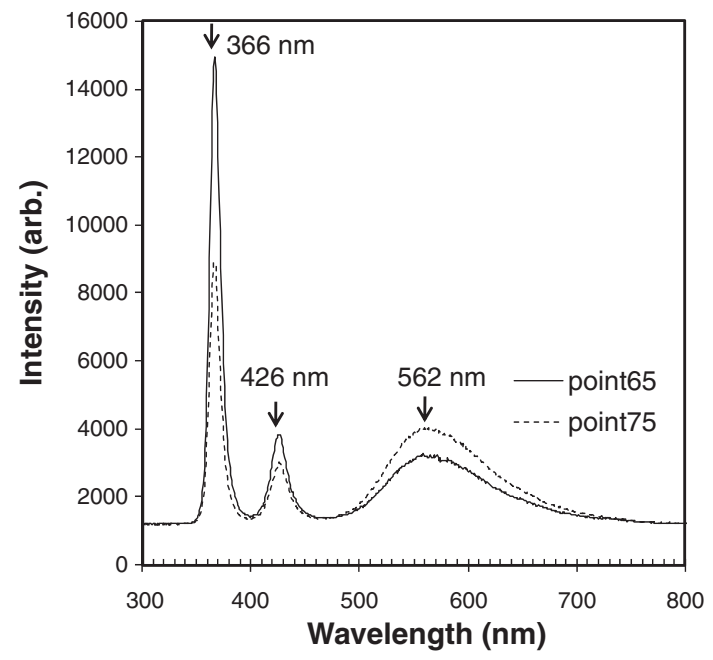

Fig. 3 Cathode luminescence spectra observed at the positions 65 and 75 in Fig. 2, as indicated by the bold arrows. Three arrows indicate the wavelengths at which the intensity mapping images in Fig. 4 were observed.

which is close to the center of a mask, exhibited a reduced yellow luminescence compared with the point 75 which is on the unmasked sapphire surface. Conversely, the luminescence from the GaN bulk and the $\mathrm{InGaN}$ well is stronger at the point 65. This trend is quite consistent with an interpretation that a number of threading dislocations in the layer on the unmasked surface provide mid-gap states that function as radiative recombination centers.

In order to clarify the spatial distribution of the intensities of those peaks, intensity mapping images were taken as shown in Fig. 4. The clearest trend is that the intensity of the yellow luminescence $(562 \mathrm{~nm})$ is small at the central part of masks. This is likely to be related to a reduced number of dislocations as described just above. Contrary to the yellow luminescence, the luminescence from the GaN band edges $(366 \mathrm{~nm})$ is stronger at the central part of masks, although the contrast is not enough in the figure. In fact, the strongest luminescence at $366 \mathrm{~nm}$ is observed at the boundary of pits on the masks, probably because the efficiency of light extraction at the boundary of the pits is larger due to the effect of 3-dimensional shape. The yellow luminescence at $562 \mathrm{~nm}$ is also enhanced slightly on the boundary of the pits similarly to the trend of $\mathrm{GaN}$, which can be explained by the same mechanism if we assume that the yellow luminescence comes from the GaN layer. The distribution of the luminescence intensity from the InGaN wells $(426 \mathrm{~nm})$ is hardly observed, except for the absence of luminescence at the pits.

Figure 5 shows the luminescence intensities for these three peaks as a function of the position that is shown in Fig. 2. Subtle variation in the intensity is more observable than the mapping images in Fig. 4. There are two trends that are overlapping in the plot;

(a) on the masks (points 9-23, 33-47, 57-68), the intensities at 366 and $427 \mathrm{~nm}$ increase while the intensity at $562 \mathrm{~nm}$ decreases;

(b) at the center of pits (points 17, 40), all the intensities at three wavelengths decreases.

The trend (a) seems to be related with the decrease in the
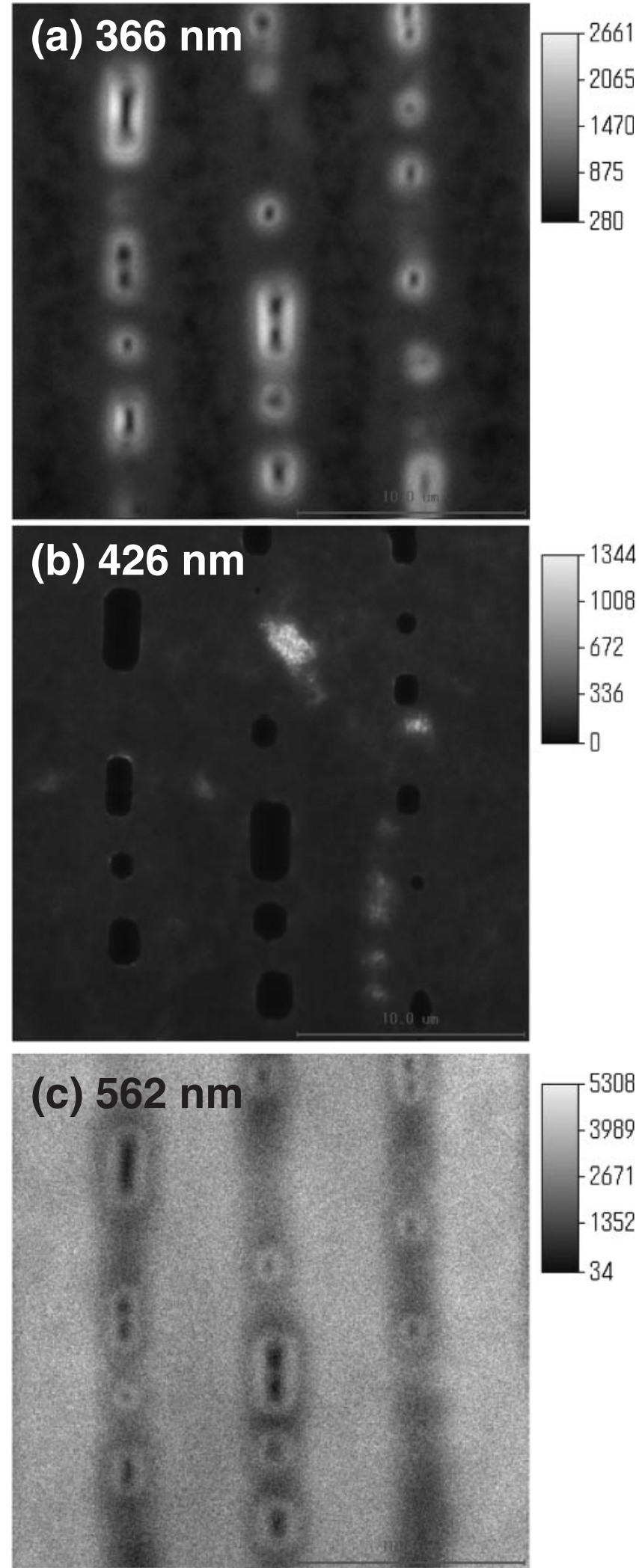

Fig. 4 Cathode luminescence intensity mapping images at (a) $366 \mathrm{~nm}$, (b) $426 \mathrm{~nm}$ and (c) $562 \mathrm{~nm}$. These wavelengths correspond to the peak wavelengths in the spectra in Fig. 3.

number of threading dislocations around the center of the masks. For the points 33-47, however, the increase in the intensity at $426 \mathrm{~nm}$ seems to be hidden behind the trend (b). The trend (b) is due to the existence of pits. At the center of 


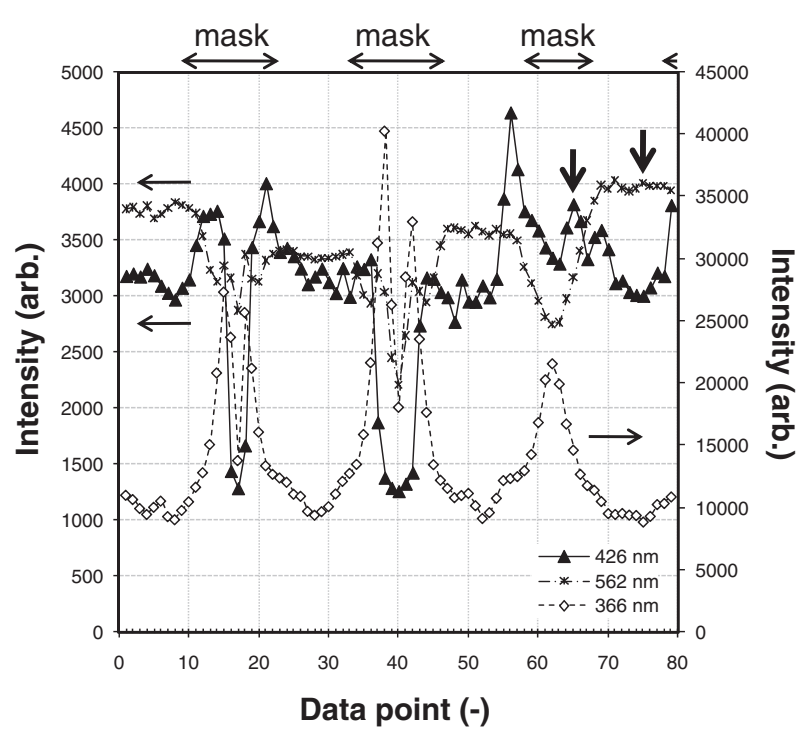

Fig. 5 Profiles of the luminescence peak intensities at $366 \mathrm{~nm}$ (open diamonds, right axis), $426 \mathrm{~nm}$ (solid triangles, left axis) and $562 \mathrm{~nm}$ (crosses, left axis) along the dot series in Fig. 2. The lines connecting the data points are just for visual assistance. The position of the masks beneath the GaN layer is presented at the top of the plot. The two bold arrows show the positions corresponding to the spectra in Fig. 3.

pits, the secondary electron signal is also weak as shown in Fig. 2. The dented shape of these pits seems to prevent the luminescence to reach the detector, resulting in the low intensity at all the wavelengths. The thickness of the InGaN wells is reported to vary significantly when the micro-facets emerge on the surface of the underlying $\mathrm{GaN},{ }^{11)}$ which shifts the luminescence wavelength from the InGaN wells. It is, therefore, natural that the luminescence at $426 \mathrm{~nm}$ disappears not only at the center of the pits but also at the whole area of the pits where micro-facets have emerged. For the points 5768 , where only one small pit exists in the vicinity of the measurement points, the trend (b) is almost negligible and we can clearly observe the trend (a).

As discussed above, the profile of the luminescence intensities indicates that the number of threading dislocations is decreased at the center of the masks, contributing to an increase in the luminescence intensities from both the GaN band edges and InGaN wells. In the present observation, the change in the luminescence from the InGaN wells is limited in its intensity. It has been reported that threading dislocations in the GaN underlayer of the InGaN/GaN MQWs often result in "V-pits", which locally alter the surface incorporation of In and Ga due to the appearance of (1011) facets and thus deforms the structure of the MQWs. ${ }^{1-3)}$ In the present study, the peak position from the InGaN wells was not affected by the existence of threading dislocations, and we did not observe such deformation of the MQWs due to the "V-pits".

\subsection{Structural analysis by STEM}

In reference to the discussion above, the structural characteristics that we have to clarify are (1) distribution of threading dislocations caused by the existence of the masks on the sapphire substrate and (2) vertical and lateral uniformity of the thicknesses of the $\mathrm{InGaN}$ wells and the

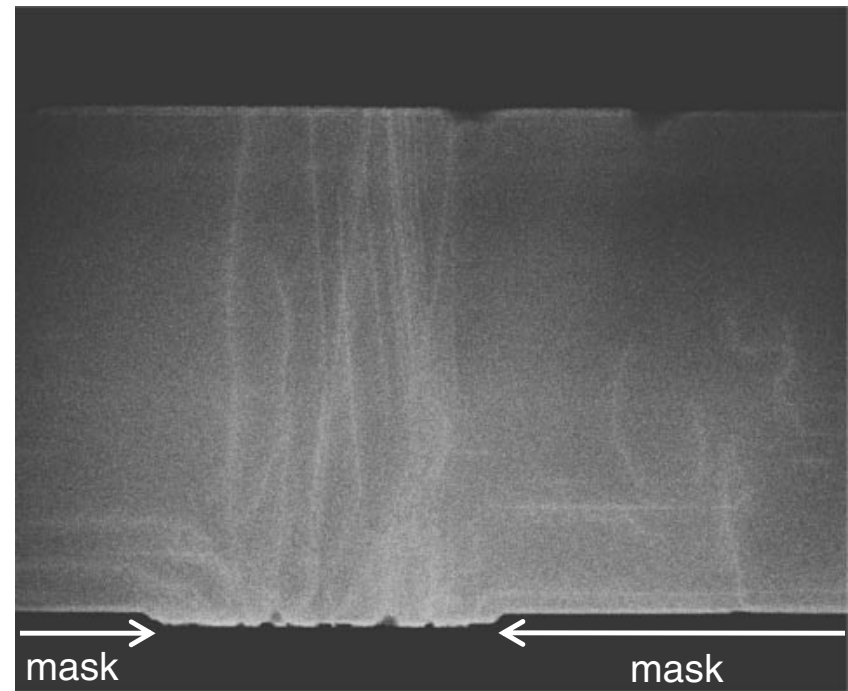

$1 \mu \mathrm{m}$

Fig. 6 Cross sectional STEM images of the GaN layer on both the masked and the unmasked sapphire surface. In these figures, white wavy lines are threading dislocations in the $\mathrm{GaN}$ layer.

GaN barriers. In order to clarify these characteristics more clearly, we have employed STEM in a thick area of the foil in order to eliminate the contrast due to the strain of samples.

In the cross sectional STEM-HAADF images in Fig. 6, the distribution of threading dislocations can be clearly seen with white lines. There are a number of threading dislocations in the GaN layer stemming from the GaN/sapphire interface. These dislocations do not propagate to the surface of the layer overriding on the masks. As a result, the GaN underlayer for the InGaN/GaN MQWs has a distinctive distribution of the number of threading dislocations; a significant number of threading dislocations on the unmasked surface while a much reduced number of dislocations on the masks. It is noted that there exists no threading dislocations in the vicinity of the surface of the layer on the masks as in the Fig. 6. It is, therefore, possible to examine the effect of threading dislocations on the optical properties and the structure of InGaN/GaN MQWs as decoupled from the effect of growth conditions, through the comparison between the MQWs on the unmasked surface and those on the masks. These findings are consistent with the trend of cathode luminescence.

Figure 7 shows the cross sectional STEM-HAADF images of InGaN/GaN MQWs both on the mask and the unmasked sapphire surface. The InGaN layers are seen in the vicinity of the surface by the lines with bright contrast due to Z-contrast effect. The thickness of the InGaN well is $3-4 \mathrm{~nm}$ and the spacing is $20 \mathrm{~nm}$ regardless of the positions, i.e., the structure of the MQWs is independent of the density of the threading dislocations in the present study. This is consistent with the luminescence peak wavelength from the InGaN wells that was independent of the measurement positions.

\section{Conclusion}

The ELO of the GaN template and the InGaN/GaN MQWs on a patterned sapphire c-plane substrate allows us to 

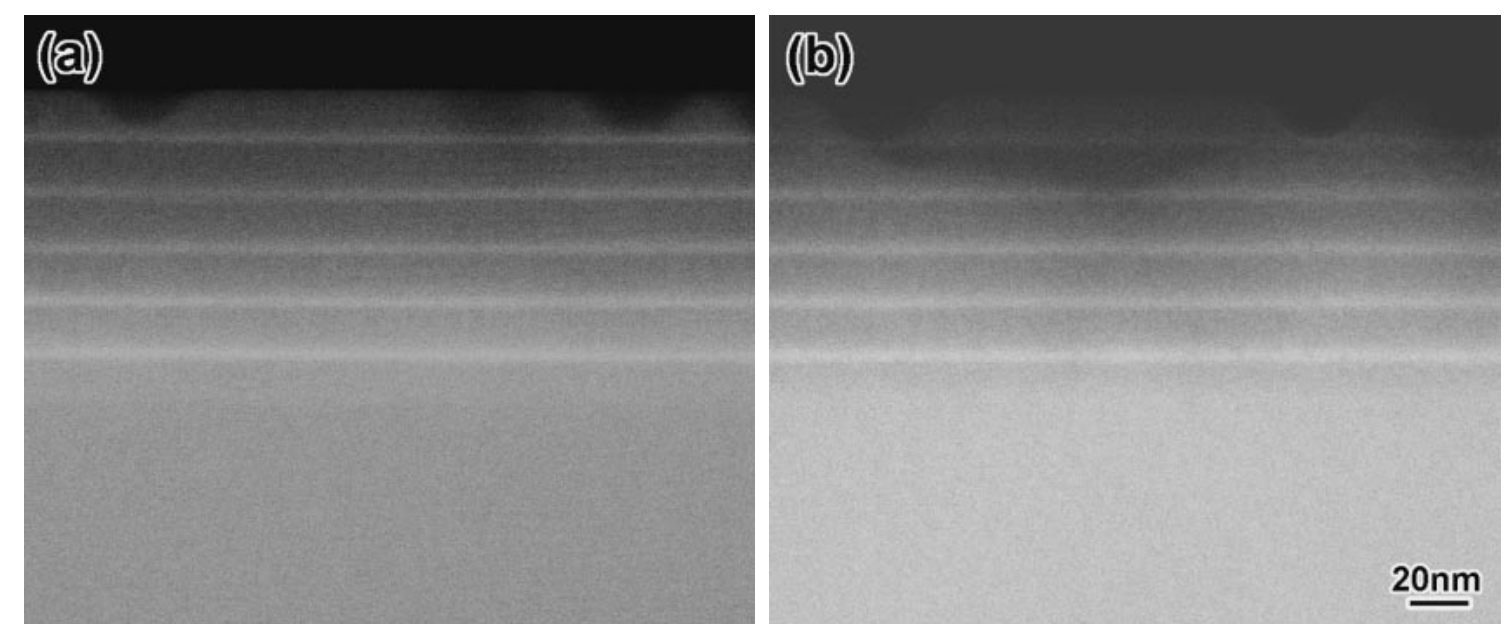

Fig. 7 Cross sectional STEM images of InGaN/GaN MQWs (a) on the unmasked sapphire surface and (b) on the mask. The bright lines in the vicinity of the surface are the InGaN wells.

examine the effect of threading dislocations on the structure and the optical properties of the InGaN well, keeping the other conditions exactly the same. On the masks, where no threading dislocation was found in the vicinity of the surface by the STEM observation, the luminescence from the InGaN well, the indium content of which was approximately $10 \%$, was stronger compared with that on the unmasked area. The "yellow luminescence", which is considered to be due to dislocations, was weaker on the masks than on the unmasked area. The luminescence wavelength from the InGaN well was independent of the position on the surface, and the structure of the well was also independent of the position as confirmed by the STEM observation. Therefore, it seems that the existence of threading dislocations just reduces the luminescence intensity through a recombination via mid-gap states and the structures of the InGaN wells is unaffected by dislocations, for the InGaN wells with relatively low indium content. This conclusion, however, can be limited to the specific range of indium content around $10 \%$, because it has been reported that the behavior of carriers in InGaN is much dependent on indium content. ${ }^{12)}$

\section{Acknowledgement}

A part of this study was supported by a Grant-in-Aid for
Scientific Research on Priority Areas "Nano Materials Science for Atomic Scale Modification 474".

\section{REFERENCES}

1) X. H. Wu, C. R. Elsass, A. Abare, M. Mack, S. Keller, P. M. Petroff, S. P. DenBaars and J. S. Speck: Appl. Phys. Lett. 72 (1998) 692.

2) H. K. Cho, J. Y. Lee, C. S. Kim, G. M. Yang, N. Sharma and C. Hamphreys: J. Crystal Growth 231 (2001) 466.

3) A. M. Yong, C. B. Soh, X. H. Zhang, S. Y. Chow and S. J. Chua: Thin Solid Films 515 (2007) 4496.

4) I. Lo, K. Y. Hsieh, S. L. Hwang, L. W. Tu, W. C. Mitchel and A. W. Saxler: Appl. Phys. Lett. 74 (1999) 2167.

5) J. Bai, T. Wang, Y. Izumi and S. Sakai: J. Crystal Growth 223 (2001) 61.

6) T. Nishinaga, T. Nakano and S. Zhang: Jpn. J. Appl. Phys. 27 (1988) L964.

7) Y. Kawaguchi, Y. Honda, H. Matsushima, M. Yamaguchi, K. Hiramatsu and N. Sawaki: Jpn. J. Appl. Phys. 37 (1998) L966.

8) T. Ogino and M. Aoki: Jpn. J. Appl. Phys. 19 (1980) 2395.

9) J. Neugebauer and C. G. Van de Walle: Appl. Phys. Lett. 69 (1996) 503.

10) R. Armitage, W. Hong, Y. Qing, H. Feick, J. Gebauer, E. R. Weber, S. Hautakangas and K. Saarinen: Appl. Phys. Lett. 82 (2003) 3457.

11) M. Ueda, K. Hayashi, T. Kondou, M. Funato, Y. Kawakami, Y. Narukawa and T. Mukai: Phys. Status Solidi C 4 (2007) 2826.

12) A. Kaneta, M. Funato and Y. Kawakami: Phys. Rev. B 78 (2008) 125317. 